\title{
Bacterial infections are associated with cardiovascular disease in Iran: a meta-analysis
}

Farzad Khademi ${ }^{1}$, Hamid Vaez ${ }^{2}$, Amir Abbas Momtazi-Borojeni ${ }^{3}$, Araz Majnooni ${ }^{4}$, Maciej Banach ${ }^{5,6}$, Amirhossein Sahebkar $7,8,9$

\author{
'Department of Microbiology, School of Medicine, Ardabil University of Medical \\ Sciences, Ardabil, Iran \\ ${ }^{2}$ Department of Microbiology, School of Medicine, Zabol University of Medical \\ Sciences, Zabol, Iran \\ ${ }^{3}$ Nanotechnology Research Center, Student Research Committee, Department \\ of Medical Biotechnology, School of Medicine, Mashhad University of Medical \\ Sciences, Mashhad, Iran \\ ${ }^{4}$ Department of Microbiology, School of Medicine, Mashhad University of Medical \\ Sciences, Mashhad, Iran \\ ${ }^{5}$ Department of Hypertension, WAM University Hospital in Lodz, Medical University \\ of Lodz, Lodz, Poland \\ 'Polish Mother's Memorial Hospital Research Institute (PMMHRI), Lodz, Poland \\ ${ }^{7}$ Biotechnology Research Center, Pharmaceutical Technology Institute, Mashhad \\ University of Medical Sciences, Mashhad, Iran \\ ${ }^{8}$ Neurogenic Inflammation Research Center, Mashhad University of Medical Sciences, \\ Mashhad, Iran \\ ${ }^{9}$ School of Pharmacy, Mashhad University of Medical Sciences, Mashhad, Iran
}

Submitted: 18 December 2017

Accepted: 3 January 2018

Arch Med Sci 2019; 15 (4): 902-911

DOI: https://doi.org/10.5114/aoms.2019.85509

Copyright (c) 2019 Termedia \& Banach

\section{Abstract}

Introduction: The present study aimed to assess the prevalence and association of various bacterial infections with cardiovascular disease (CVD) in Iran. Material and methods: An electronic search was performed using related keywords in the national and international databases up to June 30, 2017. Out of the 1807 articles found on the associations between bacterial infections and CVD, 20 relevant studies were selected for the meta-analysis.

Results: The prevalence of bacterial infections was higher in case groups compared with the control groups. Odds ratios for assessing the association between Chlamydia pneumonia infection and CVD based on PCR, IgG and IgA tests were $7.420(95 \% \mathrm{Cl}: 3.088-17.827), 3.710(95 \% \mathrm{Cl}: 1.361-10.115)$ and 2.492 (95\% Cl: $1.305-4.756)$, respectively. Moreover, the calculated odds ratio for Mycoplasma pneumonia infection was 1.815 (95\% Cl: 0.973-3.386). For Helicobacter pylori infection, odds ratios based on IgG and IgA tests were 3.160 (95\% Cl: $1.957-5.102)$ and 0.643 (95\% Cl: 0.414-0.999), respectively. Conclusions: The present meta-analysis suggested that there was a significant association between H. pylori, C. pneumonia and M. pneumonia infections and CVD in Iran. These findings confirm the potential role of bacterial infections as predisposing factors for CVD.

Key words: bacterial infection, cardiovascular disease, meta-analysis, Iran.

\section{Introduction}

Cardiovascular disease (CVD) is a major cause of death all over the world $[1,2]$. Atherosclerosis is a chronic inflammatory disease in the vas-

\author{
Corresponding author: \\ Amirhossein Sahebkar \\ PharmD, PhD \\ Department of \\ Medical Biotechnology \\ School of Medicine \\ Mashhad University \\ of Medical Science \\ 91779-48564 \\ Mashhad, Iran \\ Phone: +98 5138002288 \\ Fax: +98 5138002287 \\ E-mail:sahebkara@mums. \\ ac.ir, \\ amir_saheb2000@yahoo.com
}


cular walls $[3,4]$ that is initiated and progresses via an interplay among multiple factors including proinflammatory cytokines and acute phase proteins such as C-reactive protein (CRP) and fibrinogen [5]. Coronary artery atherosclerosis is a key risk factor in the development of acute coronary syndrome [6].

Among various established clinical and laboratory risk factors associated with the pathogenesis of CVD (e.g. diabetes, hypertension, hyperlipidemia, dyslipidemia and smoking), several lines of evidence have shown that bacterial pathogens may play a main role $[1,4,7,8]$. Bacterial infections can contribute to CVD mainly through interaction with inflammatory and immunological pathways, either directly or indirectly [1]. Infection has been found to directly impair endothelial function by circulating endotoxins, induce proliferation of smooth muscle cells and local inflammation, and activate the innate immune response [9]. On the other hand, indirect damaging effects of bacterial infections include induction of proinflammatory, hypercoagulability and atherogenic responses, oxidation of low-density lipoprotein, antigen mimicry between bacterial and host cells, induction of nutrient/vitamin malabsorption, and metabolic disruptions such as excess production of ammonia. To sum up, repeated exposure to bacterial infections leads to an excess inflammatory process leading to the provocation of immune responses that adversely affect cardiovascular risk factors such as triglycerides, high-density lipoprotein, C-reactive protein, heat shock proteins, cytokines, fibrinogen, and white blood cell count. Different bacterial species related to the risk of CVD include Helicobacter pylori, Chlamydia pneumonia, Mycoplasma pneumonia and Porphyromonas gingivalis [7-11].

In Iran, a developing country in the Middle East, atherosclerosis is a prevalent disease with a high morbidity and mortality burden [7]. The association between CVD and bacterial infections is not fully known in the Iranian population and some studies have emphasized the existence of a relationship, while others have not found any association. Therefore, we aimed to investigate the possible relationship between main bacterial infections and CVD among Iranian patients using a systematic review and meta-analysis approach.

\section{Material and methods}

\section{Search strategy}

Up to June 30, 2017, a comprehensive search of publications was performed on the relationship between bacterial infections and CVD in Iran using both Persian- and English-language databases including Scientific Information Database (www.sid.ir),
Irandoc (www.irandoc.ac.ir), Iranmedex (www.iranmedex.ir) and Magiran (www.magiran.com) as well as PubMed, MEDLINE, Scopus, Google Scholar and ISI web of knowledge. The following search terms were selected from the MeSH database: "heart diseases" OR "cardiovascular diseases" OR "coronary artery disease" OR "atherosclerosis" OR "myocardial infarction" OR "ischemic heart disease" AND "bacterial infections" OR "H. pylori" OR "C. pneumonia" OR " $P$. gingivalis" OR " $M$. pneumonia" OR "bacterial periodontal diseases" AND "Iran". Additional related articles were obtained through scanning the reference lists and hand searching. Drafting the systematic reviews and meta-analysis was based on the PRISMA checklist [12].

\section{Study eligibility criteria}

After evaluating article titles, abstracts and keywords, case-control studies were selected based on the eligibility criteria including: 1) case-control studies published in Persian and English languages, 2) assessing the relationship between bacterial infections and CVD in Iran, and 3) reporting the prevalence of CVD only in humans. Exclusion criteria were: 1) studies published in other languages, 2) articles except cross-sectional or case-control studies, 3) cross-sectional studies without a control comparator, 4) papers without full text, 5) Persian articles without an English abstract, 6) review articles, 7) duplicate studies, for which only the most recent reports were included, 8) studies presented in conferences as abstracts, 9) assessment of relationships between viral, fungal and parasitic infections with CVD, and 10) evaluated the prevalence rate of bacterial infection and CVD alone.

\section{Data collection process}

To reduce possible bias and mistakes, data extraction from each included study was performed by independent authors. Two reviewers extracted data after reviewing the title, abstract and full text of papers and the following information was then extracted and summarized in Table I [13-31]: 1) first author's surname, 2) year of the study, 3) area (city) of the study, 4) mean age for case and control groups, 5) gender (F/M) ratio for case and control groups, 6) number of patients with different types of CVD, 7) CVD type, 8) type and number of bacterial infections, and 9) bacterial infection detection techniques.

\section{Data synthesis}

Comprehensive Meta-Analysis (CMA) software version 2.2 (Biostat, Englewood, NJ, USA) was used for statistical analysis and the association between bacterial infections and CVD was expressed as odds ratio (OR) and 95\% confidence in- 


\begin{tabular}{|c|c|c|c|c|c|c|c|c|c|c|c|}
\hline \multicolumn{2}{|c|}{ 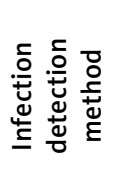 } & 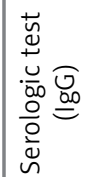 & 品容 & 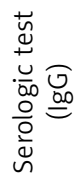 & 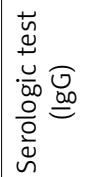 & 号崖 & 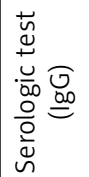 & 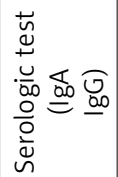 & 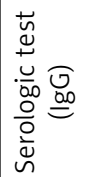 & 品 & 品 \\
\hline 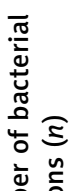 & 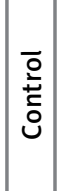 & $\begin{array}{l}\bar{z} \\
\frac{\partial}{a} \\
\bar{a} \\
\dot{1}\end{array}$ & 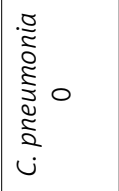 & 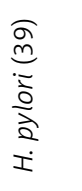 & 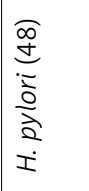 & 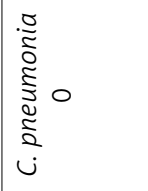 & 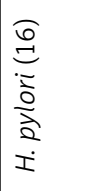 & 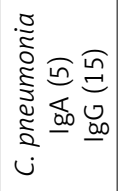 & $\begin{array}{l}\frac{\widehat{D}}{0} \\
\bar{a} \\
\frac{a}{a} \\
\dot{I}\end{array}$ & 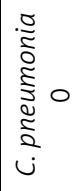 & 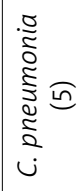 \\
\hline 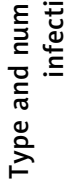 & 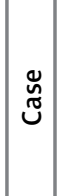 & 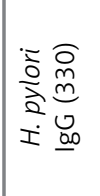 & 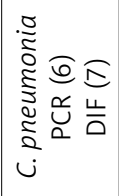 & 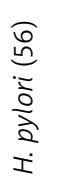 & 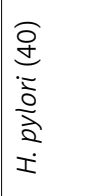 & 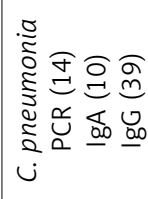 & 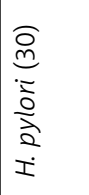 & 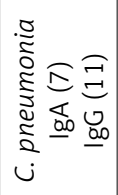 & 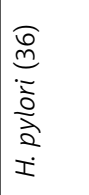 & 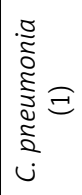 & 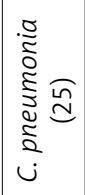 \\
\hline 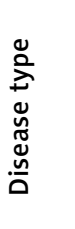 & & 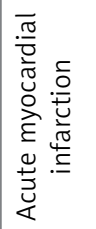 & 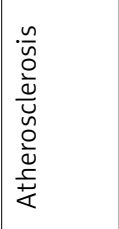 & 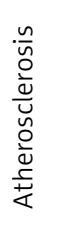 & 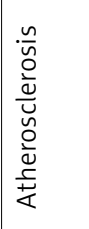 & 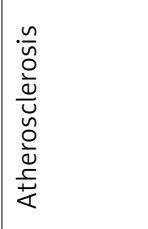 & 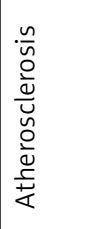 & 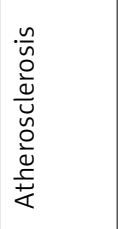 & 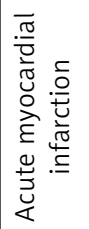 & 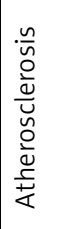 & 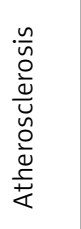 \\
\hline 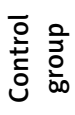 & & i & $\vec{m}$ & 8 & $\check{\infty}_{\infty}$ & $\stackrel{t}{\sim}$ & q & $\infty$ & in & ○ & $\stackrel{\sim}{\infty}$ \\
\hline 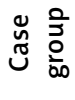 & & ঃ & $m$ & ং & 8 & मे & రิ & $\mathscr{\gamma}$ & in & ○ & $\stackrel{\sim}{\infty}$ \\
\hline$\sum_{\text {L }}$ & 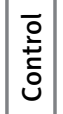 & $\begin{array}{l}\stackrel{\circ}{\stackrel{N}{\sim}} \\
\stackrel{\leftrightarrow}{\sim}\end{array}$ & $\underset{\sigma}{\stackrel{N}{\sigma}}$ & $\stackrel{\vec{m}}{\stackrel{\vec{\nu}}{\sim}}$ & 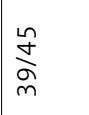 & 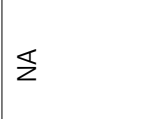 & 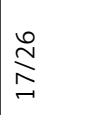 & $\underset{\substack{n \\
\infty}}{\stackrel{\infty}{\sigma}}$ & $\frac{9}{n}$ & $\frac{\pi}{z}$ & $\frac{\vec{f}}{\vec{f}}$ \\
\hline S్ల & 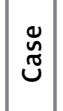 & 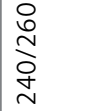 & $\underset{\Xi}{\stackrel{\Xi}{\Xi}}$ & 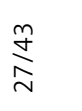 & 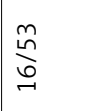 & $\stackrel{+}{m}$ & $\stackrel{\sim}{\stackrel{\sim}{\check{~}}}$ & 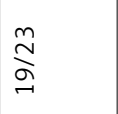 & 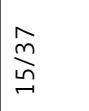 & 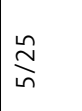 & 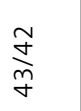 \\
\hline$\overline{\frac{n}{\pi}}$ & 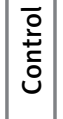 & $\stackrel{\substack{n \\
\infty \\
\infty}}{\stackrel{\infty}{+}}$ & $\stackrel{\text { Ln }}{q}$ & $\vec{b}$ & 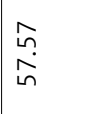 & $\frac{\pi}{z}$ & $\begin{array}{l}\text { un } \\
\text { ò } \\
\text { in }\end{array}$ & $\begin{array}{l}\vec{D} \\
0 \\
0\end{array}$ & 文 & $\frac{\pi}{z}$ & 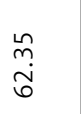 \\
\hline$\underset{\leftarrow}{\stackrel{\alpha}{0}}$ & 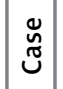 & $\vec{n}$ & ติ & ธ & 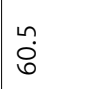 & 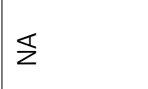 & 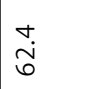 & $\begin{array}{l}\vec{\lambda} \\
\infty \\
0\end{array}$ & in & $\tilde{m}$ & $\overrightarrow{6}$ \\
\hline 忍 & & 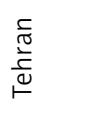 & $\frac{\frac{\sigma}{\sigma}}{\stackrel{\frac{\pi}{\sigma}}{\frac{\omega}{\omega}}}$ & 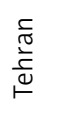 & 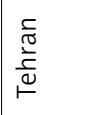 & 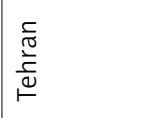 & $\begin{array}{l}\frac{c}{\pi} \\
\frac{\sqrt{\pi}}{\sqrt{\pi}} \\
\underline{\underline{w}}\end{array}$ & $\begin{array}{l}\frac{c}{\pi} \\
\frac{\pi}{\sqrt[\pi]{\pi}} \\
\underline{\underline{n}}\end{array}$ & $\begin{array}{l}\frac{\sqrt{\pi}}{\sqrt{\pi}} \\
\frac{\sqrt[\pi]{\pi}}{\underline{\pi}} \\
\underline{n}\end{array}$ & 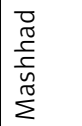 & 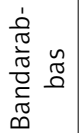 \\
\hline 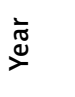 & & $\begin{array}{l}\text { 옹 } \\
\text { in }\end{array}$ & 尚 & $\begin{array}{l}\text { bे } \\
\stackrel{\circ}{\circ} \\
\stackrel{N}{0}\end{array}$ & 光 & $\overrightarrow{\grave{d}}$ & $\stackrel{\vec{d}}{\stackrel{\vec{d}}{\vec{d}}}$ & 岁 & $\begin{array}{ll}1 & \sigma \\
\infty & \Omega \\
\sigma & \sigma \\
\sigma & \sigma\end{array}$ & $\stackrel{\circ}{\stackrel{\circ}{\sim}}$ & $\overrightarrow{\stackrel{1}{\vec{c}}} \overrightarrow{\stackrel{\vec{D}}{\sim}}$ \\
\hline 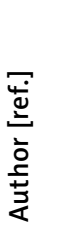 & & 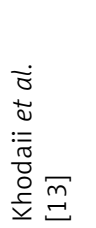 & 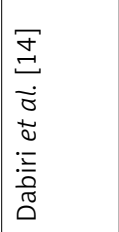 & 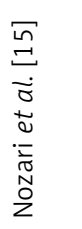 & 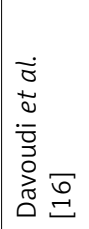 & 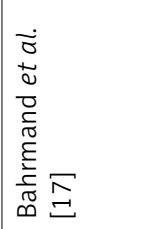 & 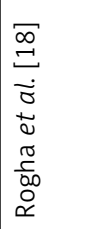 & 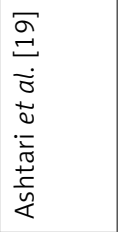 & 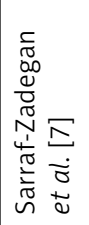 & 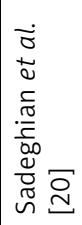 & 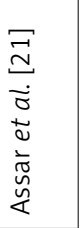 \\
\hline
\end{tabular}




\begin{tabular}{|c|c|c|c|c|c|c|c|c|c|c|c|}
\hline \multicolumn{2}{|c|}{ 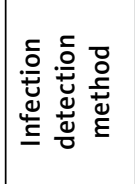 } & 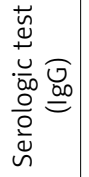 & 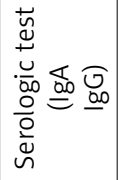 & 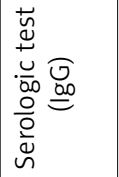 & 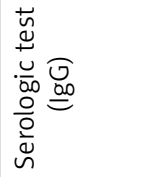 & 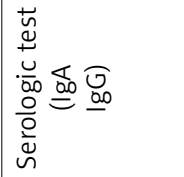 & 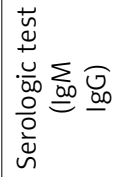 & 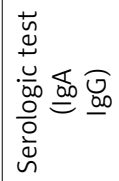 & 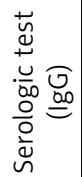 & 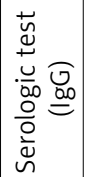 & 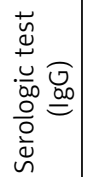 \\
\hline 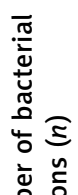 & 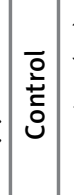 & 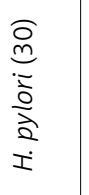 & 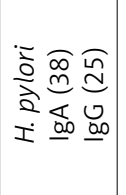 & 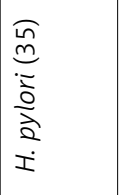 & 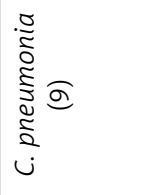 & 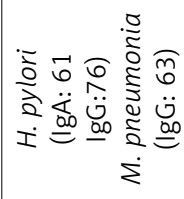 & 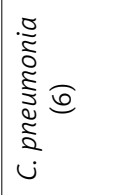 & 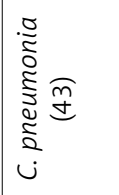 & 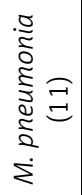 & 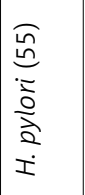 & 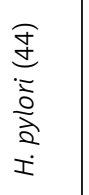 \\
\hline 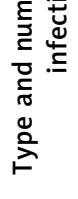 & 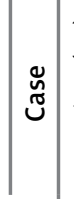 & 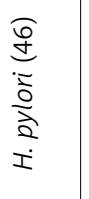 & 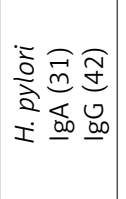 & 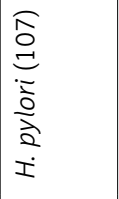 & 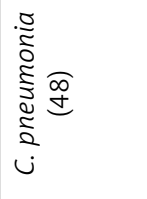 & 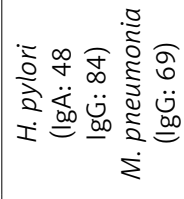 & 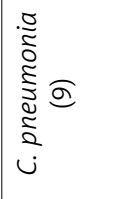 & 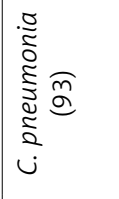 & 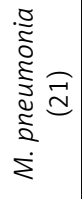 & 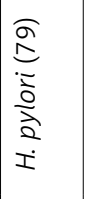 & 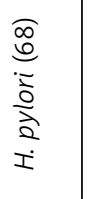 \\
\hline 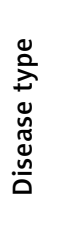 & & 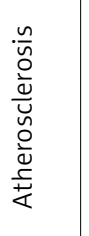 & 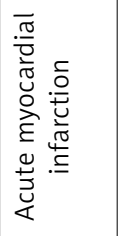 & 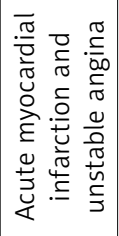 & 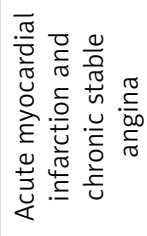 & 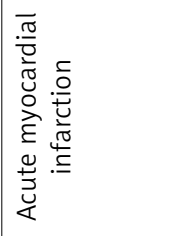 & 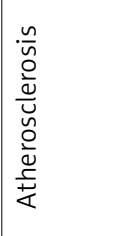 & 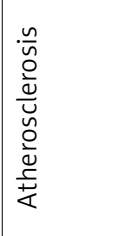 & 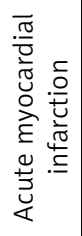 & 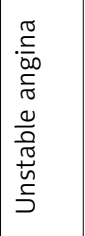 & 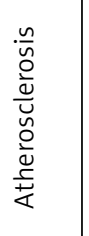 \\
\hline 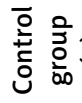 & & $\stackrel{\infty}{n}$ & $\stackrel{\infty}{\wedge}$ & 8 & $\stackrel{\text { Nे }}{\text { s. }}$ & 8 & ৪ & in & if & ๙ & $\stackrel{\curvearrowright}{\infty}$ \\
\hline 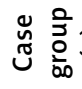 & & $\widetilde{\sigma}$ & n & $\underset{\underset{\sim}{\sim}}{\stackrel{O}{*}}$ & $\stackrel{\infty}{\infty}$ & ৪ & ৪ & Oे & 占 & ஃ & 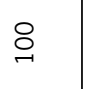 \\
\hline$\underbrace{\bar{\Sigma}}_{\underline{\underline{U}}}$ & 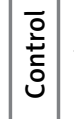 & $\underset{m}{\stackrel{D}{\sim}}$ & 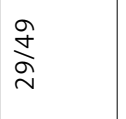 & 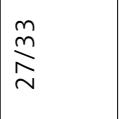 & $\stackrel{\infty}{\leftrightarrows}$ & $\begin{array}{l}\vec{b} \\
\text { సे }\end{array}$ & $\frac{\vec{f}}{\sigma}$ & 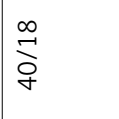 & $\underset{\stackrel{J}{\sim}}{\stackrel{\sim}{\sim}}$ & $\underset{\substack{0 \\
\text { i }}}{ }$ & $\underset{⿱}{\stackrel{N}{ }}$ \\
\hline ভ్ & $\mid \begin{array}{l}\tilde{n} \\
\tilde{J} \\
\tilde{J}\end{array}$ & $\stackrel{\substack{n \\
N}}{\sim}$ & 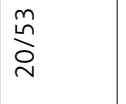 & $\frac{d}{\frac{d}{0}}$ & 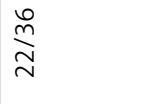 & $\underset{m}{\stackrel{n}{m}}$ & $\frac{\vec{\lambda}}{a}$ & $\begin{array}{l}\infty \\
\stackrel{0}{*} \\
\vec{\gamma}\end{array}$ & $\underset{m}{\vec{J}}$ & 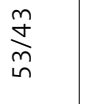 & $\frac{\partial}{\partial}$ \\
\hline $\begin{array}{l}\overline{\frac{n}{\pi}} \\
\bar{\sigma}\end{array}$ & 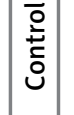 & $\ddot{n}$ & $\begin{array}{l}\text { t. } \\
\stackrel{\circ}{n}\end{array}$ & ì & in & $\begin{array}{l}\infty \\
o \\
\text { in }\end{array}$ & $\begin{array}{l}0 \\
\dot{1}\end{array}$ & in & $\overrightarrow{\vec{i}}$ & $\underset{\substack{n \\
\infty \\
\stackrel{\infty}{n}}}{ }$ & $\stackrel{\sim}{\sim}$ \\
\hline$\stackrel{80}{\alpha}$ & 离 & $\stackrel{a}{i}$ & $\begin{array}{l}\infty \\
\text { o. } \\
\text { in }\end{array}$ & 足 & in & $\begin{array}{l}\hat{n} \\
\infty \\
\infty \\
n\end{array}$ & 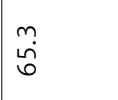 & in & 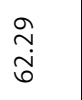 & $\begin{array}{l}\text { 文 } \\
\stackrel{\leftrightarrow}{\circ} \\
\text { in }\end{array}$ & in \\
\hline 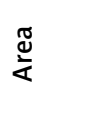 & & $\frac{\varepsilon}{\partial}$ & 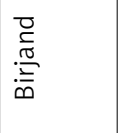 & 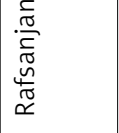 & 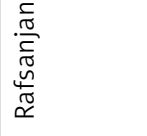 & 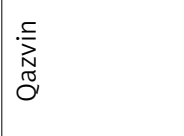 & 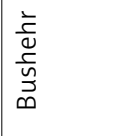 & 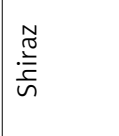 & 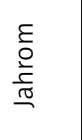 & $\sum_{\frac{\pi}{4}}^{N}$ & 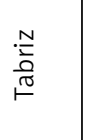 \\
\hline 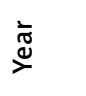 & & $\stackrel{m}{\stackrel{N}{N}}$ & $\overrightarrow{\vec{D}}$ & $\begin{array}{ll}1 & \infty \\
0 & 0 \\
0 & 0 \\
\end{array}$ & 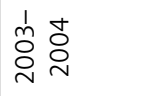 & $\stackrel{m}{\stackrel{n}{v}}$ & $\underset{\sim}{\stackrel{\vec{D}}{~}}$ & ळ్ & 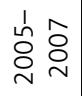 & 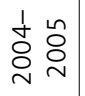 & 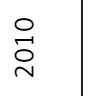 \\
\hline 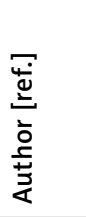 & & 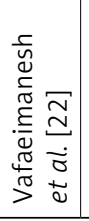 & 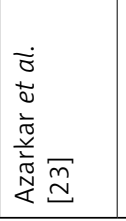 & 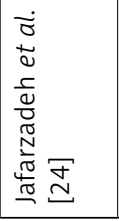 & 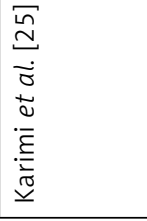 & 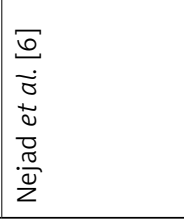 & 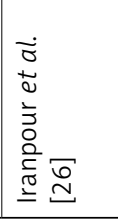 & 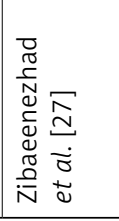 & 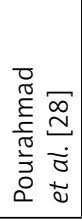 & 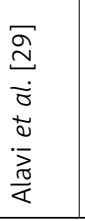 & 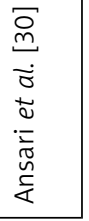 \\
\hline
\end{tabular}


terval $(95 \% \mathrm{Cl})$. Risk of publication bias was evaluated using Begg's and Egger's tests. Existence of heterogeneity across studies was determined using the Cochrane $\mathrm{Q}$ test and the inconsistency index $\left(I^{2}\right)$. In the case of small and large heterogeneity, fixed- and random-effects models were used to pool the results. In all analyses, $p<0.05$ was considered as statistically significant.

\section{Results}

\section{Study characteristics}

As shown in the flow diagram, a total of 20 casecontrol studies were obtained on the relationship between different bacterial infections and CVD in Iran up to June 30, 2017. Briefly, 1807 original articles were collected from various national and international databases. After title, abstract and full text screening, 1792 articles were omitted based on exclusion criteria. Five articles were included after assessing the references and the metaanalysis was performed on 20 selected articles. Several papers were excluded because they had no effect on meta-analysis results. These selected papers were from Tehran ( 5 studies), Isfahan (3 studies), Rafsanjan (2 studies), and Mashhad, Tabriz, Shiraz, Ahwaz, Qazvin, Qom, Bandar Abbas, Bushehr, Birjand, and Jahrom (each with 1 study) (Figure 1). Profiles of selected studies in the metaanalysis are listed in Table I [13-30]. Serological tests were the most important techniques used for the diagnosis of bacterial infections in patients with CVD in Iran. The most common type of CVD was atherosclerotic CVD.

\section{The association between C. pneumonia infection and CVD}

In Iran, a total of 8 relevant studies evaluated the association between $C$. pneumonia infection and CVD (Table I). Molecular and serological methods were the most important diagnostic tests for the detection of $C$. pneumonia infection. Based on PCR, IgG and IgA tests, C. pneumonia infection prevalence in case groups was $22.7 \%$ (46/202), $56.6 \%(200 / 353)$, and 53.6\% (110/205), respectively. However, in control groups, the positive rate of $C$. pneumonia infection on the basis of PCR, IgG and IgA tests was $2.9 \%$ (5/172), 25.7\% (73/283), and $29.2 \%$ (48/164), respectively. Odds ratios for the association between C. pneumonia infection and CVD based on PCR, IgG and IgA tests were 7.420 (95\% Cl: 3.088-17.827), $3.710(95 \% \mathrm{Cl}$ : 1.361-10.115) and 2.492 (95\% Cl: $1.305-4.756)$, respectively (Figure 2 ).

There was low heterogeneity of the included studies; therefore, we used a fixed-effect model for assessing the association between $C$. pneumonia infection and CVD on the basis of PCR $(p=0.829$,
$\left.P^{2}=0 \%\right)$ and $\operatorname{lgA}\left(p=0.480, P^{2}=0 \%\right)$. However, based on the IgG test ( $p=0.002, R^{2}=75.7 \%$ ), a random-effects model was used to demonstrate the association between infection and disease. As shown in Table II, Begg's and Egger's tests were used to evaluate publication bias of studies.

\section{The association between M. pneumonia infection and CVD}

There were only 2 studies evaluating the association between $M$. pneumonia infections and CVD in Iran. IgG-based serologic testing was the only method used to detect $M$. pneumonia infection (Table I). In the present study, the prevalence rate of $M$. pneumonia infection on the basis of the IgG test in case and control groups was $66.6 \%$ (90/135) and 54.8\% (74/135), respectively. Moreover, the calculated odds ratio for $M$. pneumonia infection was 1.815 (95\% Cl: 0.973-3.386).

A fixed-effect model was applied, due to low heterogeneity ( $p=0.252, l^{2}=23.7 \%$ ), to pool results and to evaluate the association between M. pneumonia infection and CVD on the basis of IgG (Figure 3).

\section{The association between $H$. pylori infection and CVD}

As shown in Table I, using serologic tests, the association between $H$. pylori infection and CVD was assessed in 10 articles in Iran. Based on IgG, the prevalence of $H$. pylori infection among case and control groups was $70.9 \%(918 / 1294)$ and $39.2 \%$ (476/1213), respectively. Furthermore, the calculated odds ratio was 3.160 (95\% Cl: 1.9575.102) (Figure 4 A). Based on IgA, the prevalence of $\mathrm{H}$. pylori infection was $48.4 \%$ (79/163) for case groups and 58.9\% (99/168) for control groups. The calculated odds ratio for $\mathrm{H}$. pylori infection was 0.643 (95\% Cl: 0.414-0.999) (Figure 4 B).

Finally, to pool the results of the IgG-based $H$. pylori test $\left(p<0.001, l^{2}=83 \%\right)$, a random-effects model was used, and for the IgA-based $\mathrm{H}$. pylori test $\left(p=0.427, I^{2}=0 \%\right.$ ), a fixed-effect model was used.

\section{Discussion}

The risk factors for the emergence and development of CVD are not the same in different patients [15]. More than a century ago, potential roles of infectious organisms including bacteria and viruses as potent risk factors in the induction of heart diseases were investigated in several epidemiological studies [31]. Chlamydia pneumonia, a Gram-negative and obligate intracellular bacterium, was the first proposed etiologic agent responsible for the induction of inflammation in the vessel walls of patients with CVD $[31,32]$. In Iran, the prevalence 
International and national databases (PubMed, Medline, Scopus, Google Scholar, and ISI web of knowledge, SID, Irandoc, IranMedex, and Magiran) N = 1807

1547 studies excluded due to: unrelated title, duplicate publications, unrelated topic

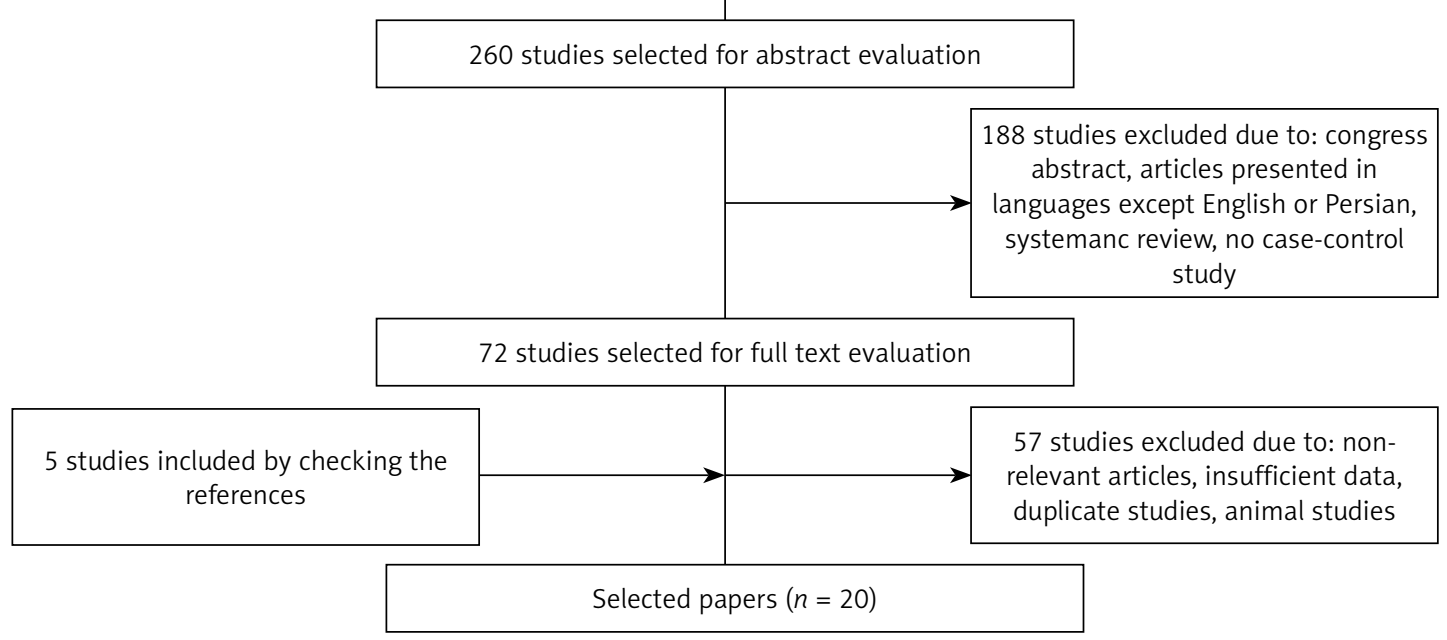

Figure 1. Flow diagram of select studies

A

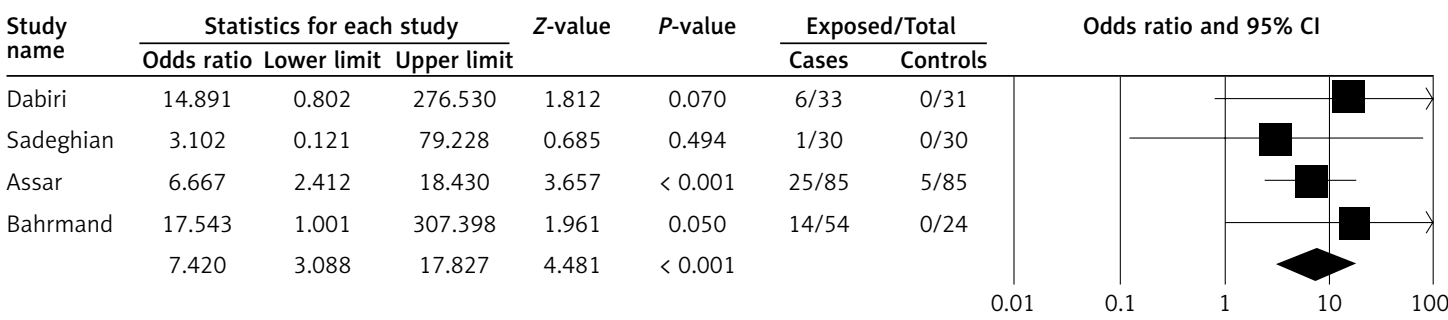

B

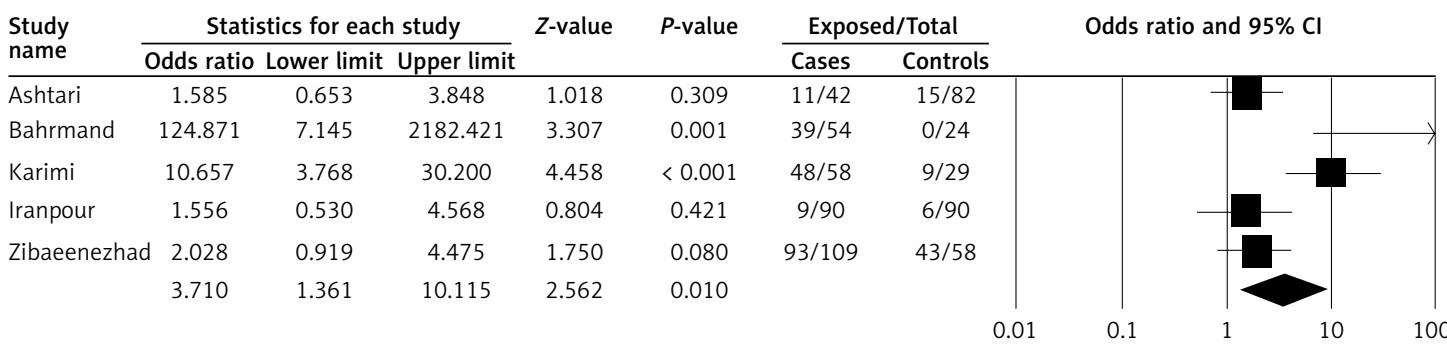

C

\begin{tabular}{|c|c|c|c|c|c|c|c|c|c|}
\hline \multirow{2}{*}{$\begin{array}{l}\text { Study } \\
\text { name }\end{array}$} & \multicolumn{3}{|c|}{ Statistics for each study } & \multirow[t]{2}{*}{$Z$-value } & \multirow[t]{2}{*}{$P$-value } & \multicolumn{2}{|c|}{ Exposed/Total } & \multicolumn{2}{|c|}{ Odds ratio and $95 \% \mathrm{Cl}$} \\
\hline & Odds ratio & Lower limit & Upper limit & & & Cases & Controls & & \\
\hline Ashtari & 3.080 & 0.914 & 10.383 & 1.814 & 0.070 & $7 / 42$ & $5 / 82$ & & \\
\hline Bahrmand & 11.362 & 0.649 & 205.875 & 1.666 & 0.096 & $10 / 54$ & $0 / 24$ & & \\
\hline \multirow[t]{2}{*}{ Zibaeenezhad } & d 2.028 & 0.919 & 4.475 & 1.750 & 0.080 & $93 / 109$ & $43 / 58$ & & \\
\hline & 2.492 & 1.305 & 4.756 & 2.768 & 0.006 & & & & \\
\hline
\end{tabular}

Figure 2. Forest plot of $C$. pneumonia infection associated with heart disease risk in Iran. A - PCR, B - IgG, C - IgAbased tests 


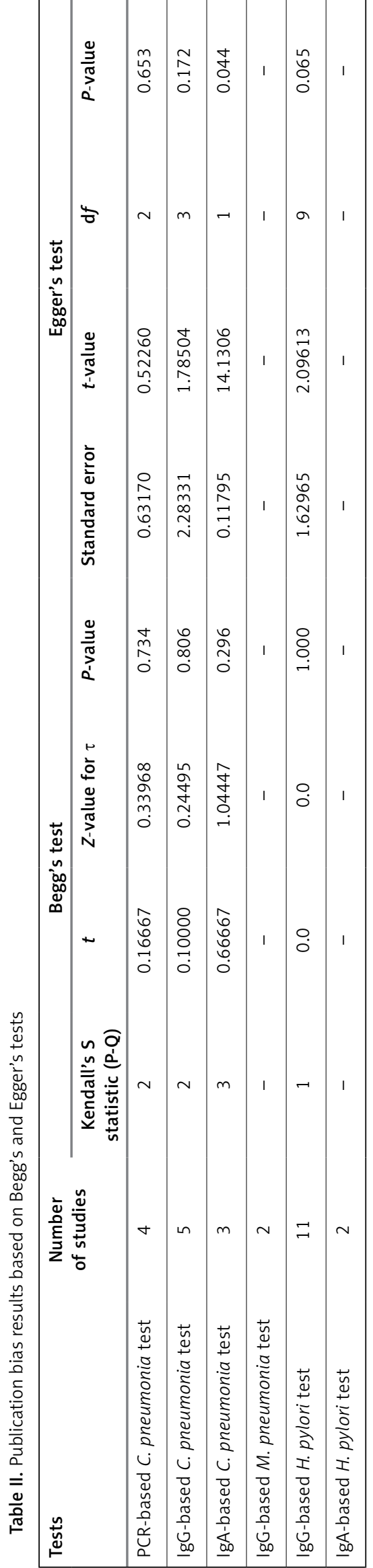

of $C$. pneumonia infection in patients with CVD is rather high [14]. However, there are confusing results about the role of $C$. pneumonia in the pathogenesis of the disease in Iran and the world. For example, Sadeghian et al. [20], Zibaeenezhad et al. [27], West et al. [33] and Apfalter et al. [34] did not find a link between C. pneumonia infection and CVD, while Dabiri et al. [14], and Sessa et al. [35] found a significant correlation. These differences in results may be due to the variations in sampling, population size, applied detection technique, ethnicity, region, and disease type [21]. In the present study, the prevalence of $C$. pneumonia infection was higher in case groups compared with control groups, as detected by PCR, IgG and IgA tests (Figures $2 \mathrm{~A}-\mathrm{C}$ ). In addition, our findings showed that the detection rate of C. pneumonia using molecular methods was lower than the serological methods in both case $(22.7 \%$ vs. $56.6 \%$ and $53.6 \%)$ and control groups (2.9\% vs. $25.7 \%$ and $29.2 \%$ ). Based on the molecular detection methods, the prevalence of $C$. pneumonia among Iranian subjects with CVD (22.7\%) was higher than in India (10\%) [36] and Turkey (15\%) [37], and lower than in Poland (27\%) [38] and Japan (62\%) [39]. Our results support the notion that there is a significant association between C. pneumonia and CVD risk in the Iranian people.

Helicobater pylori, a Gram-negative and microaerophilic bacterium, colonizes the stomach of approximately $50 \%$ of the world's population and is involved in gastritis, peptic ulcer, adenocarcinoma and MALT lymphoma [40-42]. There is inconsistent evidence on the association between H. pylori infection and CVD risk in Iran. Khodaii etal.[13] reported a significant association between H. pylori infection and CVD. In contrast, no association was found between $H$. pylori infection and CVD in a study reported by Eskandarian et al. [43]. In the present meta-analysis, we found a significant correlation between $\mathrm{H}$. pylori infection and $C V D$ risk in Iran based on the IgG test $(O R=3.160$, $p<0.001$ ). However, there was no relationship between $H$. pylori infection and CVD based on the IgA test $(O R=0.643, p=0.049)$. One reason for this inconsistency could be the low number of studies in the latter category $(n=2)$. Two studies from Poland and Germany showed a significant association between $\mathrm{H}$. pylori infection (detected according to genotypic and serological methods, respectively) and CVD risk [38, 44]. In contrast, one study from Austria did not suggest a main role for H. pylori infection in CVD [45]. The existence of these conflicting results suggests that the use of highly sensitive and specific methods is necessary to confirm the hypothetical relationship between infectious agents and the development of CVD.

In recent studies, it was suggested that the smallest pathogenic cause of atypical pneumonia 


\begin{tabular}{|c|c|c|c|c|c|c|c|c|c|c|}
\hline \multirow{2}{*}{$\begin{array}{l}\text { Study } \\
\text { name }\end{array}$} & \multicolumn{3}{|c|}{ Statistics for each study } & \multirow[t]{2}{*}{$Z$-value } & \multirow[t]{2}{*}{$P$-value } & \multicolumn{2}{|c|}{ Exposed/Total } & \multirow{2}{*}{\multicolumn{3}{|c|}{ Odds ratio and $95 \% \mathrm{Cl}$}} \\
\hline & Odds ratio & Lower limit & Upper limit & & & Cases & Controls & & & \\
\hline Pourahmad & 2.705 & 1.103 & 6.634 & 2.173 & 0.030 & $21 / 45$ & $11 / 45$ & & & \\
\hline \multirow[t]{3}{*}{ Nejad } & 1.408 & 0.724 & 2.737 & 1.009 & 0.313 & $69 / 90$ & $63 / 90$ & & & \\
\hline & 1.815 & 0.973 & 3.386 & 1.874 & 0.061 & & & & & \\
\hline & & & & & & & 0.01 & 0.1 & 10 & 100 \\
\hline
\end{tabular}

Figure 3. Forest plot of M. pneumonia infection associated with heart disease risk in Iran (lgG-based test)

A

\begin{tabular}{|c|c|c|c|c|c|c|c|c|}
\hline \multirow{2}{*}{$\begin{array}{l}\text { Study } \\
\text { name }\end{array}$} & \multicolumn{3}{|c|}{ Statistics for each study } & \multirow[t]{2}{*}{$Z$-value } & \multirow[t]{2}{*}{$P$-value } & \multicolumn{2}{|c|}{ Exposed/Total } & \multirow[t]{2}{*}{ Odds ratio and $95 \% \mathrm{Cl}$} \\
\hline & Odds ratio & Lower limit & Upper limit & & & Cases & Controls & \\
\hline Khodaii & 7.765 & 5.829 & 10.344 & 14.007 & $<0.001$ & $330 / 500$ & $100 / 500$ & \\
\hline Nozari & 2.154 & 0.977 & 4.747 & 1.903 & 0.057 & $56 / 70$ & $39 / 60$ & \\
\hline Davoudi & 1.034 & 0.543 & 1.971 & 0.103 & 0.918 & $40 / 69$ & $48 / 84$ & \\
\hline Rogha & 1.582 & 0.715 & 3.500 & 1.132 & 0.258 & $30 / 62$ & $16 / 43$ & \\
\hline Sarraf-Zadegan & an 13.219 & 5.096 & 34.292 & 5.308 & $<0.001$ & $36 / 52$ & $8 / 55$ & \\
\hline Vafaeimanesh & hh 2.683 & 1.246 & 5.780 & 2.521 & 0.012 & $46 / 62$ & $30 / 58$ & \\
\hline Azarkar & 2.872 & 1.478 & 5.582 & 3.112 & 0.002 & $42 / 73$ & $25 / 78$ & \\
\hline Jafarzadeh & 5.879 & 2.719 & 12.713 & 4.502 & $<0.001$ & $107 / 120$ & $35 / 60$ & \\
\hline Nejad & 2.579 & 0.944 & 7.049 & 1.847 & 0.065 & $84 / 90$ & $76 / 90$ & \\
\hline Alavi & 3.464 & 1.787 & 6.715 & 3.679 & $<0.001$ & $79 / 96$ & $55 / 96$ & \\
\hline \multirow[t]{2}{*}{ Ansari } & 2.173 & 1.204 & 3.924 & 2.575 & 0.010 & $68 / 100$ & $44 / 89$ & \\
\hline & 3.160 & 1.957 & 5.102 & 4.708 & $<0.001$ & & & \\
\hline
\end{tabular}

B

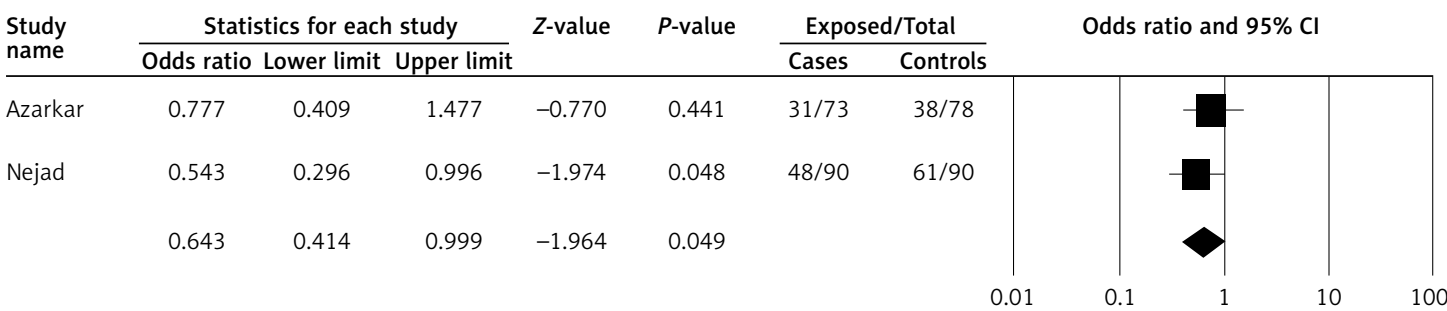

Figure 4. Forest plot of $\mathrm{H}$. pylori infections associated with heart disease risk in Iran. A - IgG, B - IgA-based tests

in human is M. pneumonia, which is a common respiratory tract microorganism related to CVD risk either alone or along with other risk factors [46]. However, in Iran, only 2 studies assessed this possible risk using serological tests (Table I). In the present study, the obtained odds ratio for $M$. pneumonia infection among case groups with CVD was 1.815 . It suggests that $M$. pneumonia infection may be a risk factor for CVD. Similar finding were reported in Saudi Arabia [46], Japan [47], the Netherlands [48] and Germany [44]. Inconsistently with our results, the role of $M$. pneumonia infection in the etiopathogenesis of CVD has been refuted in some studies [38, 45]. Various sensitivity and specificity values for different diagnostic tests for detecting $M$. pneumonia infection in patients with CVD may be an important reason for discrepancies in the results obtained in different studies [48]. In this meta-analysis, no study was found on the association between $P$. gingivalis infection and resulting bacterial periodontal infections and CVD risk in Iran. Finally, we recommend evaluation of the association between cardiovascular risk factors and biomarkers such as plasma low- and high-density lipoprotein (LDL and HDL) and vitamin $\mathrm{D}$ status with various bacterial infections that predispose to CVD [49-51].

In conclusion, the present systematic review focused on the association between different bacterial infections and CVD risk in Iran. Our metaanalysis revealed that $H$. pylori, C. pneumonia and $M$. pneumonia infections could be considered as potential risk factors for CVD in Iran. These findings are worthwhile to confirm the role of bacterial infections as predisposing factors for CVD and also explore the bacterial species that have a stronger association with CVD. Based on the present findings, efficient and timely eradication of $H$. pylori, C. pneumonia and M. pneumonia infections could be a useful strategy to reduce the burden of CVD. This study was limited in several ways including heterogeneity of diagnostic tests used for the detection of infections, and lack of a complete spectrum of studied bacterial species in relation to CVD. Furthermore, our analysis was limited to Iran, and a global investigation will be crucial for generalization of the findings. Based on the mentioned limitations, additional studies 
are needed to explain the difference between obtained results on the basis of various bacterial infection detection methods. Finally, the association between other bacterial infections and CVD risk in Iran merits further investigations.

\section{Conflict of interest}

The authors declare no conflict of interest.

\section{References}

1. Hogas S, Bilha SC, Branisteanu D, et al. Potential novel biomarkers of cardiovascular dysfunction and disease: cardiotrophin-1, adipokines and galectin-3. Arch Med Sci 2017; 13: 897-913.

2. Campbell LA, Kuo C. Chlamydia pneumonia-an infectious risk factor for atherosclerosis? Nature Rev Microbiol 2004; 2: 23-32.

3. Hoseini Z, Sepahvand F, Rashidi B, Sahebkar A, Masoudifar A, Mirzaei H. NLRP3 inflammasome: its regulation and involvement in atherosclerosis. J Cell Physiol 2018; 233: 2116-32.

4. Rezaee-Zavareh MS, Tohidi M, Sabouri A, RamezaniBinabaj M, Sadeghi-Ghahrodi M, Einollahi B. Infectious and coronary artery disease. ARYA Atheroscler 2016; 12 41-9.

5. Khater ES, Aziz BAA. Helicobacter pylori infection as a risk factor for coronary artery disease. Int J Trop Dis Health 2016; 15: 1-8.

6. Nejad ND, Mostafaei L, Jahanbakhshi F, Rashvand F, Alipour MH, Saroukhani MR. The correlation of infectious and inherent agents with acute myocardial infarction in patients of teaching hospitals in Qazvin in 2013. Open Access Library J 2015; 2: e1656.

7. Sarraf-Zadegan N, Amiri M, Maghsoudloo S. Helicobacter pylori relation to acute myocardial infarction in an Iranian sample. Coron Health Care 2001; 5: 202-7.

8. Liu F, Wang Y, Xu J, Liu F, Hu R, Deng H. Effects of Porphyromonas gingivalis lipopolysaccharide on the expression of key genes involved in cholesterol metabolism in macrophages. Arch Med Sci 2016; 12: 959-67.

9. Banach M, Markuszewski L, Zasłonka J, Grzegorczyk J, Okoński P, Jegier B. The role of infection in the pathogenesis of atherosclerosis. Przegl Epidemiol 2004; 58: 671-6.

10. Matusiak A, Chałubiński M, Broncel M, et al. Putative consequences of exposure to Helicobacter pylori infection in patients with coronary heart disease in terms of humoral immune response and inflammation. Arch Med Sci 2016; 12: 45-54.

11. Binder CJ, Hörkkö S, Dewan A, et al. Pneumococcal vaccination decreases atherosclerotic lesion formation: molecular mimicry between Streptococcus pneumonia and oxidized LDL. Nat Med 2003; 9: 736-43.

12. Liberati A, Altman DG, Tetzlaff J, et al. The PRISMA statement for reporting systematic reviews and metaanalyses of studies that evaluate health care interventions: explanation and elaboration. PLoS Med 2009; 6: e1000100.

13. Khodaii Z, Vakili H, Ghaderian SMH, Najar RA, Panah AST. Association of Helicobacter pylori infection with acute myocardial infarction. Coron Artery Dis 2011; 22: 6-11.

14. Dabiri H, Rezadehbashi M, Badami N, et al. Detection of Chlamydia pneumonia in atherosclerotic plaques of patients in Tehran, Iran. Jpn J Infect Dis 2009; 62: 195-7.
15. Akiash N, Nozari Y, Ebrahimi Daryani N, Abdollahi A. Association between Helicobacter pylori infection and atherosclerotic coronary artery disease. Iran J Pathol 2009; 4: 1-4.

16. Davoudi S, Omran A, Boroumand M, Rahimian N, Saadat S. Association between Helicobacter pylori and coronary artery disease. Open Med 2011; 6: 107-12.

17. Bahrmand AR, Bahadori M, Hossaini A, et al. Chlamydia pneumonia DNA is more frequent in advanced than in mild atherosclerosis lesions. Scand J Infect Dis 2004; 36: 119-23.

18. Rogha M, Nikvarz M, Pourmoghaddas Z, Shirneshan K, Dadkhah D, Pourmoghaddas M. Is Helicobacter pylori infection a risk factor for coronary heart disease? ARYA Atheroscler 2012; 8: 5-8.

19. Ashtari F, Saberi A, Shayegannejad V, Khosravi A, Sherkat R, Khosravi E. Association between Chlamydia pneumonia infection and carotid atherosclerotic plaques. J Res Med Sci 2007; 12: 165-71.

20. Sadeghian MH, Yazdi SAT, Ayatollahi H, et al. Is there any relationship between Chlamydophila pneumonia and coronary atherosclerosis among Iranians? Niger Med J 2013; 54: 40-4.

21. Assar O, Nejatizadeh A, Dehghan F, Kargar M, Zolghadri N. Association of Chlamydia pneumonia infection with atherosclerotic plaque formation. Glob J Health Sci 2016; 8: 260-7.

22. Vafaeimanesh J, Hejazi SF, Damanpak V, Vahedian M, Sattari M, Seyyed majidi M. Association of Helicobacter pylori infection with coronary artery disease: is Helicobacter pylori a risk factor? Sci World J 2014; 2014: 1-6.

23. Azarkar Z, Jafarnejad M, Sharifzadeh G. The relationship between Helicobacter pylori infection and myocardial infarction. Caspian J Intern Med 2011; 2: 222-5.

24. Jafarzadeh A, Nemati M, Tahmasbi M, Ahmadi P, Rezayati $M$, Sayadi $A$. The association between infection burden in Iranian patients with acute myocardial infarction and unstable angina. Acta Med Indones 2011; 43: 105-11.

25. Karimi M, Nough H, Lotfi M, et al. Evaluation of anti Chlamydia pneumonia antibody in patients with coronary artery disease. Indian J Community Med 2006; 31: 34-8.

26. Iranpour D, Abbasi F, Mirzayee K, Mohammadzadeh N, Raeisi A, Najafi A. Evaluation of association between Chlamydophila pneumonia and atherosclerotic plaques in coronary artery disease patients with abnormal angiography. Health 2014; 6: 2505-9.

27. Zibaeenezhad MJ, Amanat A, Alborzi A, Obudi A. Relation of Chlamydia pneumonia infection to documented coronary artery disease in Shiraz, Southern Iran. Angiology 2005; 56: 43-8.

28. Pourahmad M, Jahromy AS, Shojaei M. Association of Mycoplasma pneumonia infection with myocardial infarction. Am J Immunol 2009; 5: 84-8.

29. Alavi SM, Adel SMH, Rajabzadeh A. Relationship between the serologic status of Helicobacter pylori with the presence of unstable angina. Pak J Med Sci 2008; 24: 29-32.

30. Khadem Ansari MH, Rasmi Y, Manafi M, Rahimipour A, Ghadermarzi E. The evaluation of Helicobacter pylori infection and cardiovascular disease risk factors with atherosclerosis. Urmia Med J 2010; 21: 17-23.

31. Izadi M, Fazel M, Sharubandi SH, et al. Helicobacter species in the atherosclerotic plaques of patients with coronary artery disease. Cardiovasc Pathol 2012; 21: 307-11.

32. Joshi R, Khandelwal B, Joshi D, Gupta OP. Chlamydophila pneumonia infection and cardiovascular disease. N Am J Med Sci 2013; 5: 169. 
33. West SK, Kohlhepp SJ, Jin R, Gleaves CA, Stamm W, Gilbert DN. Detection of circulating Chlamydophila pneumonia in patients with coronary artery disease and healthy control subjects. Clin Infect Dis 2009; 48: 560-7.

34. Apfalter P, Barousch W, Nehr M, Willinger B, Rotter M, Hirschl AM. No evidence of involvement of Chlamydia pneumonia in severe cerebrovascular atherosclerosis by means of quantitative real-time polymerase chain reaction. Stroke 2004; 35: 2024-8.

35. Sessa R, Di Pietro M, Schiavoni G, et al. Measurement of Chlamydia pneumonia bacterial load in peripheral blood mononuclear cells may be helpful to assess the state of chlamydial infection in patients with carotid atherosclerotic disease. Atherosclerosis 2007; 195: e224-30.

36. Campbell LA, Kuo C, Grayston JT. Chlamydia pneumonia and cardiovascular disease. Emerg Infect Dis 1998; 4: 571-8.

37. Iriz E, Cirak MY, Engin ED, et al. Effects of atypical pneumonia agents on progression of atherosclerosis and acute coronary syndrome. Acta Cardiol 2007; 62: 593-8.

38. Reszka E, Jegier B, Wasowicz W, et al. Detection of infectious agents by polymerase chain reaction in human aortic wall. Cardiovasc Pathol 2008; 17: 297-302.

39. Ouchi K, Fujii B, Kudo S, et al. Chlamydia pneumonia in atherosclerotic and nonatherosclerotic tissue. J Infect Dis 2000; 181: 441-3.

40. Khademi F, Faghri J, Poursina F, et al.. Resistance pattern of Helicobacter pylori strains to clarithromycin, metronidazole and amoxicillin in Isfahan, Iran. J Res Med Sci 2013; 18: 1056-60.

41. Khademi F, Faghri J, Moghim S, et al. The study of mutation in 235 rRNA resistance gene of Helicobacter pylori to clarithromycin in patients with gastrointestinal disorders in Isfahan - Iran. Adv Biomed Res 2014; 3: 98.

42. Khademi F, Poursina F, Hosseini E, Akbari M, Ghasemian Safaei H. Helicobacter pylori in Iran: a systematic review on the antibiotic resistance. Iran J Basic Med Sci 2015; 18: 2-7.

43. Eskandarian R, Ghorbani R, Shiyasi M, Momeni B, Hajifathalian K, Madani M. Prognostic role of Helicobacter pylori infection in acute coronary syndrome: a prospective cohort study: cardiovascular topics. Cardiovasc J Afr 2012; 23: 131-5.

44. Espinola-Klein C, Rupprecht HJ, Blankenberg S, et al. Impact of infectious burden on extent and long-term prognosis of atherosclerosis. Circulation 2002; 105: 15-21.

45. Weiss TW, Kvakan H, Kaun C, et al. No evidence for a direct role of Helicobacter pylori and Mycoplasma pneumonia in carotid artery atherosclerosis. J Clin Pathol 2006; 59: 1186-90.

46. Abdul-Wahab O, Alsheri A, Assiri A, Almasswary A, Benahmed F. Association of Mycoplasma pneumonia infection with ischemic heart diseases. Am J Immun 2012; 8: 117-22.

47. Momiyama Y, Ohmori R, Taniguchi H, Nakamura H, Ohsuzu F. Association of Mycoplasma pneumonia infection with coronary artery disease and its interaction with chlamydial infection. Atherosclerosis 2004; 176: 139-44.

48. Maraha B, Berg H, Scheffer GJ, et al. Correlation between detection methods of Chlamydia pneumoniae in atherosclerotic and non-atherosclerotic tissues. Diagn Microbiol Infect Dis 2001; 39: 139-43.

49. Khademi F, Momtazi-Borojeni AA, Reiner Ž, Banach M, Al-Rasadi KA, Sahebkar A. PCSK9 and infection: a potentially useful or dangerous association? J Cell Physiol 2018; 233: 2920-7.
50. Sonmez A, Nikolic D, Dogru T, et al. Low- and high-density lipoprotein subclasses in subjects with nonalcoholic fatty liver disease. J Clin Lipidol 2015; 9: 576-82.

51. Căpuşa C, Stefan G, Stancu S, llyes A, Dorobanțu N, Mircescu G. Subclinical cardiovascular disease markers and vitamin $\mathrm{D}$ deficiency in non-dialysis chronic kidney disease patients. Arch Med Sci 2016; 12: 1015-22. 